\title{
Downlink Joint Base-station Assignment and Packet Scheduling Algorithm for Cellular CDMA/TDMA Networks
}

\author{
Keivan Navaie and Halim Yanikomeroglu \\ Broadband Communications and Wireless Systems (BCWS) Centre \\ Department of Systems and Computer Engineering, Carleton University, Ottawa, Canada \\ Email: \{keivan,halim\}@sce.carleton.ca
}

\begin{abstract}
In this paper using a utility-based approach, downlink packet transmission in a CDMA/TDMA cellular network is formulated as an optimization problem. A utility function corresponds to each packet served by a base-station that is an increasing function of the packet experienced delay and the channel gain, and a decreasing function of the base-station load. Unlike previous works, in this paper, the optimization objective is to maximize the total network utility instead of the base-station utility. We show that this optimization results in joint base-station assignment and packet scheduling. Therefore, in addition to multi-user diversity, the proposed method also exploits multi-access-point diversity and soft capacity. A polynomial time heuristic algorithm is then proposed to solve the optimization problem. Simulation results indicate a significant performance improvement in terms of packet-drop-ratio and achieved throughput.
\end{abstract}

\section{INTRODUCTION}

In wireless communications, multi-user diversity gain is achieved via scheduling the transmission when corresponding time varying channel capacity happens to be at (or near) its peak. In such techniques delay tolerance of data services, alongside the radio resource fluctuations in the physical layer is opportunistically utilized to provide efficient resource allocation in data services (see e.g. [1]). This approach has been employed in high-speed downlink standards for cellular communications such as HSDPA [2] and 1xEV-DV [3], where it is usually assumed that there is a network functionality that assigns a base-station (BS) for transmission to each user.

In order to facilitate the BS assignment, each BS broadcasts a pilot signal. A user may receive the pilot signals of various BSs with the corresponding bit-energy to the interferenceplus-noise-spectral-density ratios $\left(E_{c} / I_{0}\right)$ greater than a performance threshold. For each user, its active-set is the set of BSs having acceptable channel quality to the user. The BS with the highest $E_{c} / I_{0}$ is then assigned to the user to function as the server [4]; time-scale of the execution of this procedure is usually very larger than that of packet scheduling. In cellular networks, specifically in the dense areas with small-size cells, a large number of users are able to receive pilot signals with acceptable quality from more than one BSs (e.g., up to $40 \%$ of UMTS users are expected to be in soft-hand-off condition, thus they have more than one BSs in their active-sets [4]).

Usually in cellular networks, the time-scale of the packet scheduling is smaller than that of the BS assignment. Therefore, it is quite likely that, at some point, due to channel temporal variations and/or user mobility, the channel between a user and a BS in its active set, will be better than that between that user and its assigned BS. This "better channel" (if and when it exists) can be used for more efficient transmission. Consequently, conventional BS assignment methods are not able to exploit multi-access-point diversity. The independent time variations of the channels between a user and the BSs in its active set introduce a type of diversity which we refer to as multi-access-point diversity [5]. To exploit multi-accesspoint diversity, in $1 \mathrm{xEV}-\mathrm{DV}$ a fast cell selection mechanism is proposed in which a BS assignment method based on the pilot signal strength is carried out in smaller time-scale than that of the conventional methods [3].

Furthermore, the conventional BS assignment as well as the fast cell selection method do not consider the traffic load of the BSs in the network. Consequently, such mechanisms may assign a user to an overloaded BS that causes larger packet transmission delays. In such cases, although there are available and unused resources in the neighboring $\mathrm{BS}(\mathrm{s})$, the users that can be served by another BS (however with lower bit rates) should be kept waiting in the queue of the assigned BS. This unused network resource in the neighboring BSs, which can be exploited, is referred to as soft-capacity [4].

In this paper, we propose a joint BS assignment and packet scheduling method which, besides the multi-user diversity, also exploits multi-access-point diversity and soft-capacity. We consider a cellular CDMA/TDMA data network. This system uses the throughput optimal time-domain scheduling to exploit multi-user-diversity in which, over each time interval, the total BS transmit power is allocated to a single user and the rest of the users are kept inactive.

We utilize the utility-based framework which is developed based on [6], in [5] and [7] for optimal downlink resource allocation in CDMA/TDMA networks. In this framework, corresponding to each packet waiting in the system queue and served by a BS, we consider a utility function that is a function of the BS load, the corresponding wireless channel quality, and the packet experienced delay. The summation of the utilities of all served packets at each time, the total network utility, is then considered as the network performance indicator. Our main objective is to maximize the total network utility.

The existing utility-based resource allocation approaches such as those presented in [8], [9], [10] and [11] only focus on maximizing the total utility of the individual BS. Such 
maximization can exploit multi-user diversity but it is not able to exploit multi-access-point diversity and soft-capacity which require a network-wide optimization. In this paper, unlike previous works, maximization of the total network utility is considered. It is shown that this maximization results in joint BS assignment and packet scheduling.

We further demonstrate that the joint BS assignment and packet scheduling can be mapped into a 0-1 multidimensional, multiple-choice knapsack problem (0-1 MMKP) that is NP-hard. To tackle the computational complexity issue, we propose a novel heuristic algorithm with polynomial time computational complexity. The proposed method can be implemented in a Radio Network Controller (RNC) which controls a set of BSs. A similar approach has been used in our previous works [12], [13] to formulated the power allocation problem in the downlink of a pure CDMA cellular system.

Joint BS assignment and resource control for the downlink is considered in [9] and [14] as well. However, in both of these methods, separate BS assignment and resource allocation algorithms are proposed. These algorithms are then combined in a sequential manner for more efficient resource utilization. The distinguishing feature of the proposed method, compared to the previous works in [9] and [14], is that it gives in one shot, both the packets scheduled for transmission and the corresponding BSs assigned to transmit those packets based on a network-wide knowledge. This exploits multi-user and multiaccess-point diversities as well as soft-capacity. Simulation results also indicate a significant improvement in the packetdrop ratio and the network throughput.

\section{SySTEM MODEL}

We consider the downlink of a cellular CDMA/TDMA data network which uses time-domain scheduling. The time $t$ is slotted with each time-slot containing $M$ frames. The length of each time-slot is $T_{w}$ seconds and the length of each frame is $T_{f}$ seconds, therefore $T_{w}=M . T_{f}$. In time-domain scheduling, within each frame, the total available power of BS $j$, denoted by $P_{j}$, is allocated to a single user while the rest of the users are kept inactive. The value of $T_{w}$ is chosen so that the channel fluctuation within this time-slot is negligible (i.e., $T_{w}$ is less than the coherence time of the channel). Data traffics are packetized into fixed $L$-bit packets.

Each packet is transmitted in an integer multiple of a frame. Each packet $i$ has a maximum delay tolerance of $D_{i} \cdot T_{w}$ seconds. We assume that $D_{i} \gg 1$ and that there are $N(t)$ packets in the system waiting to be transmitted in time $t$. For packet $i, d_{i}(t)$ denotes the total delay incurred from its arrival to the system until $t$, normalized to $T_{w}$. A packet $i$ with $d_{i}(t)>D_{i}$ is dropped and removed from the system.

Let $\mathcal{B}$ be the set of $B$ BSs in the network. Each BS transmits its own pilot signal. For packet $i$, an active-set $\mathcal{A}_{i}(t)$ is defined as the set of BSs in $\mathcal{B}$ which can be assigned to the corresponding destined user of packet $i$ as a transmitter:

$$
\mathcal{A}_{i}(t)=\left\{j \mid j \in \mathcal{B}, \gamma_{i j}(t) \geq \gamma_{\min }\right\},
$$

where $\gamma_{i j}(t)$ is the bit-energy to the interference-plus-noisespectral-density ratio of the pilot channel $\left(E_{c} / I_{0}\right)$ of $\mathrm{BS} j$ received by the corresponding destined user of packet $i . \gamma_{\min }$ is the minimum required $E_{c} / I_{0}$. The number of BSs in $\mathcal{A}_{i}(t)$, denoted by $N_{\mathcal{A}_{i}}(t)$, is usually limited by the air interface protocol; we denote this maximum by $N_{\mathcal{A}}$. If $N_{\mathcal{A}_{i}}(t) \geq 1$ then packet $i$ can potentially be transmitted to its destined user by one of those $N_{\mathcal{A}_{i}}(t)$ BSs.

For each packet $i$ at time $t$, corresponding to its destined user and to each BS $j$ in its active-set $\mathcal{A}_{i}(t), \rho_{i j}(t)$ denotes the average bit-energy to the interference-plus-noise-spectraldensity $E_{b} / I_{0}$. Parameter $\rho_{i j}(t)$ is a performance metric that indicates the quality of the wireless link between the destined user of packet $i$ and BS $j$ :

$$
\rho_{i j}(t)=\frac{W}{L} \frac{P_{j}(t) g_{i j}(t)}{I_{i j}(t)} T_{i j}(t),
$$

where $W$ is the chip rate, $P_{j}(t)$ is the transmit power of $\mathrm{BS}$ $j, g_{i j}(t)$ is the stable channel gain between $\mathrm{BS} j$ and the corresponding destined user of packet $i, I_{i j}(t)$ is the received interference-plus-noise power by the corresponding destined user of packet $i$ when served by BS $j, T_{i j}(t)=\frac{L}{r_{i j}(t)} \leq$ $T_{w}$ is the time extent in which packet $i$ is transmitted to its corresponding destined user by BS $j$ using time-domain scheduling ${ }^{1}$, where $r_{i j}(t)$ is the bit-rate of the channel between BS $j$ and the corresponding destined user of packet $i$ in timeslot $t$. In CDMA systems, variable bit-rates are implemented by using the orthogonal variable spreading factor technique [4]. For a given $\rho_{i j}(t), T_{i j}(t)$ can be determined from (2). We denote the number of air interface frames that should be allocated to packet $i$ in $\mathrm{BS} j$ as

$$
m_{i j}(t)=\left\lceil\frac{T_{i j}(t)}{T_{f}}\right\rceil,
$$

where $\lceil x\rceil$ denotes the rounding up to the smallest integer greater or equal to $x$.

We define the assignment matrix $\mathbf{b}(t)=$ $\left\{b_{i j}(t)\right\}_{N(t) \times(B+1)}$, with $i=1, \ldots, N(t)$, and $j=0, \ldots, B$, where $b_{i j}(t)$ is the assignment indicator at time $t$; that is $b_{i j}(t)=1$, if BS $j$ is assigned to the corresponding destined user of packet $i$, and $b_{i j}(t)=0$, otherwise. Note that, for the BSs which are not in $\mathcal{A}_{i}(t), b_{i j}(t)=0$. We also define $j=0$ corresponding to a virtual NULL BS. For every packet $i$, assignment of the corresponding destined user to the NULL BS $\left(b_{i 0}(t)=1\right)$ at time $t$ means that no transmission is scheduled for packet $i$, in this case we set $m_{i 0}=0$. Hereafter, we consider the NULL BS in the active set of all packets.

For a $\mathrm{BS} j$, we define $0<\eta_{j}(t) \leq 1$ as an indicator of the BS downlink load at time $t$. In a cellular CDMA network, for a BS $j, \eta_{j}(t)$ is a function of the allocated power to the users as well as the maximum BS transmit power (see, e.g., [4] and [15]). A larger value of $\eta_{j}(t)$ indicates a more loaded BS.

\footnotetext{
${ }^{1}$ Note that in (2) for the BSs in the $\mathcal{A}_{i}(t), \rho_{i j}(t)$ is greater than a system performance threshold to ensure that $T_{i j}(t) \leq T_{w}$.
} 


\section{A. Utility Function}

Unlike previous works, in this paper we define the utility function for each packet, not for users. Utility function serves as an optimization objective for packet transmission. It can be used to optimize radio resource allocation to build a bridge among different service and network parameters in different layers. A utility function $u_{i j}(t)$ is associated with packet $i$ served by BS $j$. The utility function indicates the "profit" earned by the network as a result of transmitting packet $i$ from BS $j$ at time $t$. The earned profit modelled by the utility function provides a priority metric for a packet served by a BS, which means the larger the value of the utility function, the higher the priority of transmitting the corresponding packet. The utility function $u_{i j}(t)$ is defined as a function of the corresponding wireless channel quality (captured by $m_{i j}(t)$ ), packets' experienced delay until time $t$ (captured by $d_{i}(t)$ ) and the corresponding BS load condition (captured by $\eta_{j}(t)$ ):

$$
u_{i j}(t) \triangleq \begin{cases}\Theta\left(d_{i}(t), m_{i j}(t), \eta_{j}(t)\right) & \text { if } j \neq 0 \& j \in \mathcal{A}_{i}(t), \\ 0 & \text { otherwise. }\end{cases}
$$

Consider two packets in the network queue waiting to be transmitted. To maximize network performance, the utility function, as a metric for the transmission priority, would be an increasing function of channel quality. Therefore, the packet destined to a user with better channel quality is given a higher transmission priority. On the other hand, the utility function should be a decreasing function of the experienced delay to manage the delay-throughout trade-off by weighing the priority of the packet with more experienced delay. Moreover, transmission from a lightly loaded BS would have a higher priority. Thus, the utility function is a decreasing function of the BS load. Therefore, $\Theta($.$) is a decreasing function of m_{i j}(t)$ and $\eta_{j}(t)$, and an increasing function of $d_{i}(t)$.

\section{Optimal Downlink PACKet Transmission}

In this paper, we define $Q(\mathbf{b} ; t)$ as the total profit earned by the network by serving the packets at each time $t$ :

$$
Q(\mathbf{b} ; t) \triangleq \sum_{i=1}^{N(t)} \sum_{j=0}^{B} u_{i j}(t) b_{i j}(t) .
$$

The optimization objective is to find an assignment matrix $\mathbf{b}(t)$ so that the total network utility $Q(\mathbf{b} ; t)$ is maximized:

\section{Problem $\mathcal{O}$ :}

$$
\begin{aligned}
\max _{\mathbf{b}(t)} & Q(\mathbf{b} ; t), \\
\text { s.t. } & \sum_{i=1}^{N(t)} m_{i j}(t) b_{i j}(t) \leq M, \quad j=1, \ldots, B, \\
& \sum_{j=0}^{B} b_{i j}(t)=1, \quad i=1, \ldots, N(t), \\
& b_{i j}(t) \in\{0,1\},
\end{aligned}
$$

where (7) indicates the network downlink resource constraints, and (8)-(9) indicate that each user should be served by only one BS. The total transmission time for packet $i$ at $\mathrm{BS} j$ is $m_{i j}(t) \cdot T_{f}$ seconds. If $b_{i 0}(t)=1$, then packet $i$ cannot be served at this time-slot.

The output of Problem $\mathcal{O}$ is $\mathbf{b}(t)$, therefore, it performs joint BS assignment and packet scheduling. Problem $\mathcal{O}$ can easily be extended to the cases such as which considered in [16], where data transmission can be performed on multiple orthogonal channels each with time-domain scheduling. Also, for the case of a given BS assignment, Problem $\mathcal{O}$ results in maximizing the total BS utility. In this case the output would be a scheduling discipline similar to those given in [1] and [17] in which at each time a packet with the highest utility function is transmitted, thus only multi-user diversity is exploited.

Here, we show that Problem $\mathcal{O}$ is a $0-1$ Multi-Dimensional, Multiple-Choice Knapsack Problem (0-1 MMKP). Since Problem $\mathcal{O}$ is solved for each time-slot, hereafter we drop the time index $t$ for brevity.

Definition (0-1 MMKP) [18]: Consider a knapsack with $B$ distinct resources, represented by $\left(M_{1}, M_{2}, \ldots, M_{B}\right)$. Assume that there are $N$ groups, indexed by $i$, each with $K_{i}$ items. Each item $j$ of a group $i$ has a particular value $u_{i j}$ and requires $B$ distinct resources, represented by the vector $\left(m_{i j 1}, m_{i j 2} \ldots, m_{i j B}\right)$. The objective of the MMKP is to select one item from each group to maximize the total value of the collected items subject to $B$ resource constraints.

Proposition 1: Optimal downlink packet transmission formulated in Problem $\mathcal{O}$ is a $0-1$ MMKP.

Proof: We consider the active-set of each packet $i, \mathcal{A}_{i}$, as a group which is associated with $K_{i}=N_{\mathcal{A}_{i}}+1 \mathrm{BSs}$ as items. Note that $\mathcal{A}_{i}$ includes NULL BS as well. The value of each item in $\mathcal{A}_{i}$ is the corresponding utility function $u_{i j}$. The required resource, $m_{i j k}=m_{i j}$ if $j=k$, and $m_{i j k}=0$, otherwise, where $m_{i j}$ is defined in (3). The objective of the optimal downlink packet transmission in Problem $\mathcal{O}$ is to select exactly one BS (i.e., item) from each group (i.e., the active set of each packet) to maximize the total network utility, subject to resource constraints. For the case that the selected BS for a packet is NULL, it is not scheduled for transmission during the current time-slot. Therefore, the downlink resource allocation in (6) is a 0-1 MMKP.

Since 0-1 MMKP is NP-complete [18], the computational complexity of finding the exact solution is very high thus not reasonable in practice. The alternative is to use heuristic approaches such as those in [13] and [18]. In Section IV, we propose a novel polynomial time heuristic algorithm for the optimal downlink packet transmission problem.

\section{Heuristic Algorithm}

The proposed heuristic algorithm is based on Lagrange Multipliers Theorem presented in [19] where it is shown that the solution of the unconstrained optimization problem

$$
\max \left\{\sum_{i=1}^{N} \sum_{j=1}^{N_{\mathcal{A}}+1}\left(u_{i j}-\lambda_{j} m_{i j}\right) b_{i j}\right\}
$$

is the solution of the constraint optimization problem $\mathcal{O}$, with

$$
b_{i j}^{*}= \begin{cases}1 & \text { if } u_{i j}-\lambda_{j} m_{i j}>0 \\ 0 & \text { otherwise. }\end{cases}
$$


Among the solutions in (11), we should find the one for which (8) is satisfied. Obviously this solution is an optimal solution. Therefore, regardless of the constraints in (8), Lagrange Multipliers Theorem is valid for the optimal downlink packet transmission, and the constraints in (8) only restrict the possible choices for $b_{i j}$. Consequently, if the multipliers $\lambda_{j}$ are known, Problem $\mathcal{O}$ can easily be solved. If these multipliers are computed so that the terms $M-\sum_{i=1}^{N} m_{i j} b_{i j}^{*}$ are nonnegative, the solution satisfies the resource constraints in (7) and is feasible; the solution is optimal if

$$
\sum_{j=0}^{B} \lambda_{j}\left(M-\sum_{i=1}^{N} b_{i j}^{*} m_{i j}\right)=0 .
$$

\section{A. The Heuristic Algorithm}

The proposed heuristic algorithm uses the Lagrange Multiplier approach as a basis for the approximation of the optimization solution. The algorithm has two main procedures which are executed consequently: the Main Body and the Solution Improvement (Fig. 1 and Fig. 2, respectively).

The algorithm starts in the Main Body by setting the Lagrange multipliers to zero in (s1.1) and normalizing the required time-slots in $(\mathrm{s} 1.2)$. Then in $(\mathrm{s} 1.3)$ each packet $i$ is assigned to a $\mathrm{BS} a(i)$ (i.e., $b_{i a(i)} \leftarrow 1$ ) in $\mathcal{A}_{i}$, where $a(i)=\arg \max u_{i j}$. Note that so far only one BS is assigned to a user, therefore, the conditions in (8) and (12) are satisfied. However, the resource constraint (7) might be violated; if so, the initial BS assignments are adjusted in the while loop until the resource constraints in (7) hold.

To adjust the initial BS assignments, the most offending BS constraint violation $j^{*}$, determined in (s1.5), is repeatedly improved in the rest of the Main Body: in (s1.6) we consider the packets whose assigned BSs are $j^{*}$. For each BS $j$ in the active-set of these packets, we then compute the increase of the Lagrange multiplier $\lambda_{j^{*}}$, denoted by $\Delta_{i j}$, resulting from exchanging the previously assigned BS $j^{*}$ by another BS in $\mathcal{A}_{i}$. Among those users whose $j^{*}$ is in their active set, we choose the user $I^{*}$ and the corresponding BS $J^{*}$ in its active set, so that the corresponding exchange causes the least increase of multiplier $\lambda_{j^{*}}$, i.e., $\min \Delta_{i j}$. In (s1.7) the exchange is done and the corresponding parameters are updated accordingly. This new assignment minimizes the gap between the optimal solution characterized by (12) and the previous assignment. The while loop in the Main Body is repeated for each packet until a BS has been assigned and (7) is satisfied (i.e., $\pi_{j} \leq 1$ ).

After the completion of the Main Body, there might be some available unused resources in the BSs. The remaining resources are utilized in the Solution Improvement algorithm (Fig. 2). In this algorithm, each BS $j$ in the active-set of every packet $i$ is checked against the currently assigned BS of that packet, i.e., $a(i)$. In (s2.1) we obtain the amount of increase in the achieved utility, denoted by $\mu_{i j}$, caused by the new assignment of $\mathrm{BS} j$, while making sure that the resource constraints are not violated. This condition is verified using the if-else statement in (s2.1). Then, in

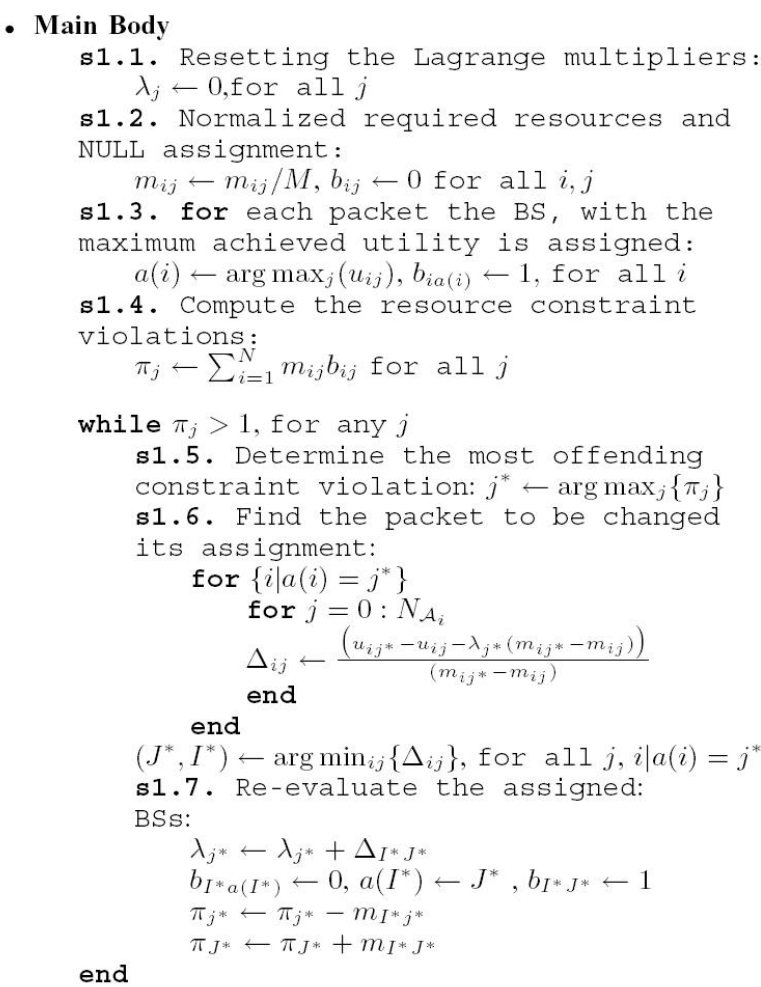

Fig. 1. The Main Body of the algorithm.

(s2.2) user $I^{\prime}$ is selected among all users, so that replacing its previously assigned BS by a new BS $J^{\prime}$ causes the largest increase in the total achieved utility, i.e., $\max \mu_{i j}$. In (s2.3) the exchange is done and the corresponding parameters are updated accordingly. The while loop is repeated until no more exchanges are possible. The resulting assignments are then considered as the solutions of the optimal downlink packet transmission.

\section{B. Implementation and Computational Complexity}

The optimization is performed in an RNC which controls a number of BSs in the network. In cases where some of the BSs in the active set of a user are controlled by different RNCs, signalling between the RNCs would be required.

The inputs of the proposed algorithm are $u_{i j}(t)$ and $m_{i j}(t)$, and the output is the assignment matrix, $\mathbf{b}(t)$. At time $t$, each user measures the pilot channel $E_{c} / I_{0}$ and finds the BSs with an acceptable pilot signal quality (i.e., $E_{c} / I_{0} \geq \gamma_{\min }$ ). For each packet $i$, the corresponding BSs with acceptable pilot signal strength in the destined user are then considered as the active-set of packet $i, \mathcal{A}_{i}(t)$. The corresponding channel qualities $\rho_{i j}(t)$ are measured and sent to the RNC by the users. Packet experienced delay, $d_{i}(t)$, is also available in the RNC. Therefore, $u_{i j}(t)$ and $m_{i j}(t)$ are obtainable. The optimal assignment matrix is then found using the proposed algorithm. The BS assignments and schedulings can be communicated to the corresponding users through signalling channels. 


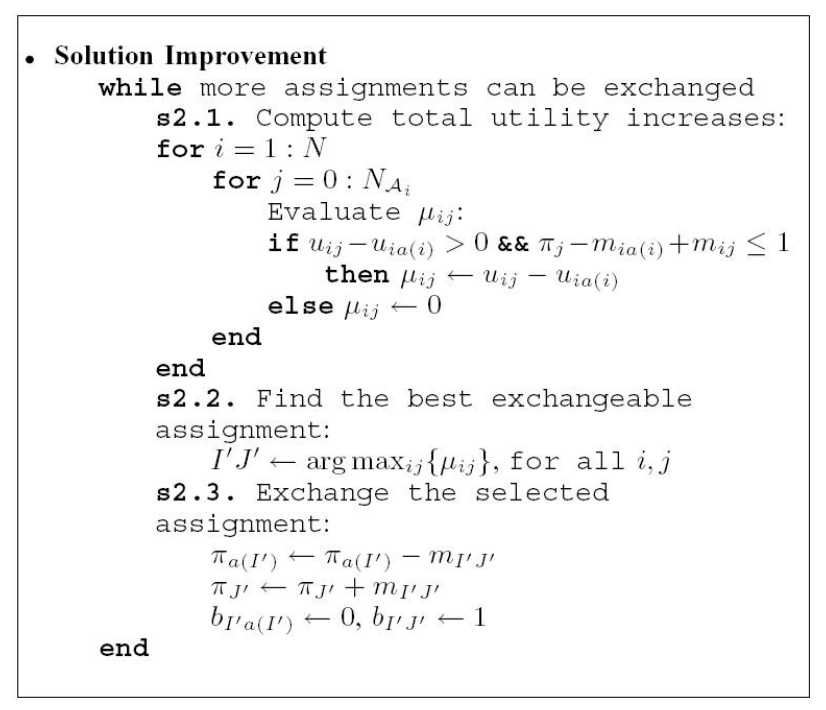

Fig. 2. Solution improvement algorithm.

The following proposition indicates that the proposed algorithm has a polynomial-time computational complexity.

Proposition 2: The heuristic algorithm in subsection IV-A has a maximum computational complexity of $O\left(N^{2} N_{\mathcal{A}}^{3}\right)$.

Proof: In the Main Body, (s1.1) has the complexity order of $O(B)$, and (s1.2)-(s1.4) have the complexity order of $O\left(N N_{\mathcal{A}}\right)$. In the while loop, (s1.5) and (s1.7) have the complexity order of $O(B)$ and $O(1)$, respectively. In (s1.6) for each of $N$ users, there are at most $N_{\mathcal{A}}$ non-selected BSs in the corresponding active-set, thus for each user the maximum complexity order is $O\left(N_{\mathcal{A}}\right)$. There is one iteration for each BS in the active-set of each user resulting in the total complexity order of $O\left(N N_{\mathcal{A}}^{2}\right)$ for (s1.6). In every iteration of (s1.6), one assigned BS is removed from one user, thus, in the worst case the while loop in the Main Body is executed $N N_{\mathcal{A}}$ times. Therefore, the overall complexity order for the execution of the while loop of the Main Body is $O\left(N^{2} N_{\mathcal{A}}^{3}\right)$.

In the Solution Improvement, the complexity order of (s2.2) and (s2.3) are $O\left(N N_{\mathcal{A}}\right)$ and $O(1)$, respectively. In (s2.1) for each of $N$ users, at most $N_{\mathcal{A}}$ non-selected BSs are in the corresponding active-set so that the total utility increase should be computed for them. Each computation has a complexity of $O\left(N_{\mathcal{A}}\right)$. There is one iteration for each BS in the active-set of each user, resulting in the complexity order of $O\left(N N_{\mathcal{A}}^{2}\right)$ for (s2.1). Since for each user there can be, at most, $N_{\mathcal{A}}$ BSs in its active-set which could have higher utility than the assigned BS, the outer while loop of the solution improvement algorithm is at most executed $N N_{\mathcal{A}}$ times. This gives an overall complexity of $O\left(N^{2} N_{\mathcal{A}}^{3}\right)$ for the solution improvement. Thus, the overall computational complexity is $O\left(N^{2} N_{\mathcal{A}}^{3}\right)$.

\section{Simulations}

The simulated cellular network is a two-tier hexagonal cell configuration consisting of 19 cells $(B=19)$; the wrap-around technique is also employed. The number of active users in the network is $N_{u}$. The simulation parameters are presented
TABLE I

Simulation PARAMETERS

\begin{tabular}{|l|c|}
\hline Parameter & Value \\
\hline \hline Physical layer & Based on UMTS \\
\hline Cell radius & $1000 \mathrm{~m}$ \\
\hline BSs transmit power & $10 \mathrm{~W}$ \\
\hline Max. No. of BSs in the active set $\left(N_{\mathcal{A}}\right)$ & 4 \\
\hline Standard deviation of log-normal fading & $8 \mathrm{~dB}$ \\
\hline Thermal noise density & $-174.0 \mathrm{dBm} / \mathrm{Hz}$ \\
\hline Propagation loss exponent & $1 \mathrm{~ms}$ \\
\hline Frame length $\left(T_{f}\right)$ & 100 \\
\hline Number of frames in each time-slot $(M)$ & $16,32,64,144,384 \mathrm{~kb} / \mathrm{s}$ \\
\hline Available bit-rates $\left(r_{i j}\right)$ & $-13 \mathrm{~dB}$ \\
\hline Required $E_{c} / I_{0}\left(\gamma_{\min }\right)$ & \\
\hline
\end{tabular}

in Table I. The pilot channel powers are adjusted so that $40 \%$ of the users receive the pilot channel of more than one BS with an acceptable quality. A non-uniform user spatial distribution is expressed by a non-uniformity factor, $\mu_{D}$, so that $\left(1-\mu_{D}\right) N_{u}$ users are distributed uniformly and the rest of them are distributed within randomly located hot-spots. For each user, fixed length packets are generated by a Poisson arrival process with an average rate of $\Lambda$ packets per second.

For a packet $i$ transmitted by a BS $j$ in time-slot $t$, we define the following utility function for $j \neq 0$ :

$$
u_{i j}(t) \triangleq \frac{1-\eta_{j}^{2}(t)}{m_{i j}(t)} \exp \left(d_{i}(t)-\bar{d}(t)\right),
$$

and $u_{i 0}=0$, where $\bar{d}(t)=\frac{1}{N(t)} \sum_{i} d_{i}(t)$ is the average delay. In (13), a packet is given a large utility when either the corresponding destined user experiences a good channel condition or a bad relative delay status. The utility function (13) also weights more for taking service from the lightly loaded BSs. Note that different utility functions can be designed to satisfy various design objectives.

For comparison, we consider three different systems. In System I (the benchmark case for comparison), the BS assignment is based on the pilot signal strength so that the BS with the highest $E_{c} / I_{0}$ is assigned as the transmission server to the user. Packets are transmitted on a first-come-first-serve basis using a time-domain scheduling scheme. In System II, the BS assignment is similar to that of System I; however, packets are scheduled so that the total utility of each cell is maximized. Therefore, in System II only multi-user diversity is exploited. Finally, System III uses the proposed joint BS assignment and packet scheduling heuristic algorithm.

We study the effect of the proposed joint BS assignment and packet scheduling algorithm on the packet-drop-ratio (PDR). PDR is defined as the probability of not transmitting a packet within its maximum tolerable delay $\left(D_{i} \cdot T_{w}\right)$. The maximum tolerable delay is assumed to be 1000 milliseconds. Fig. 3 illustrates the PDR versus packet arrival rate, $\Lambda$, for the above network setting, with $N_{u}=190\left(N_{u} / B=10\right)$. Two different cases of user spatial distributions $\left(\mu_{D}=0.2\right.$ and $\left.\mu_{D}=0.5\right)$ are considered; simulations are executed 10,000 times. It is observed from Fig. 3 that System III yields in better better PDR performance. When highly non-uniform user distribution $\left(\mu_{D}=0.5\right)$ is considered, higher PDR improvement is 


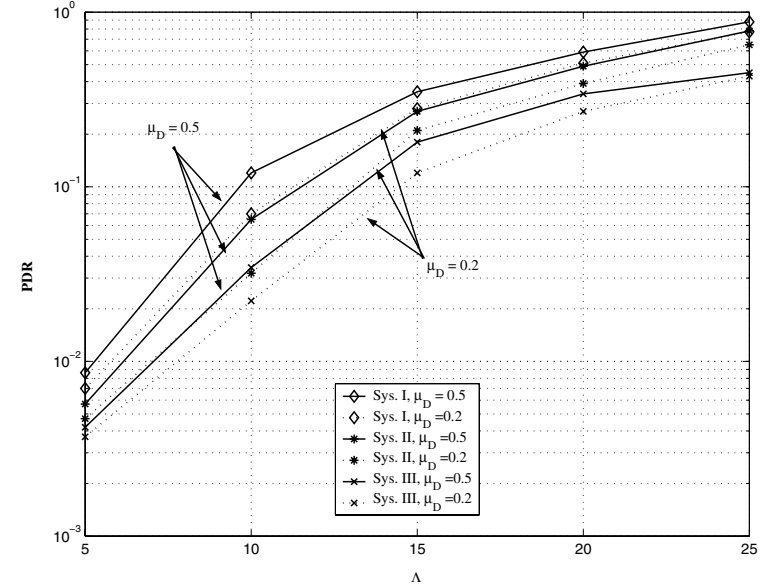

Fig. 3. Packet drop ratio (PDR) vs. packet arrival rate for $\mu_{D}=0.2,0.5$.

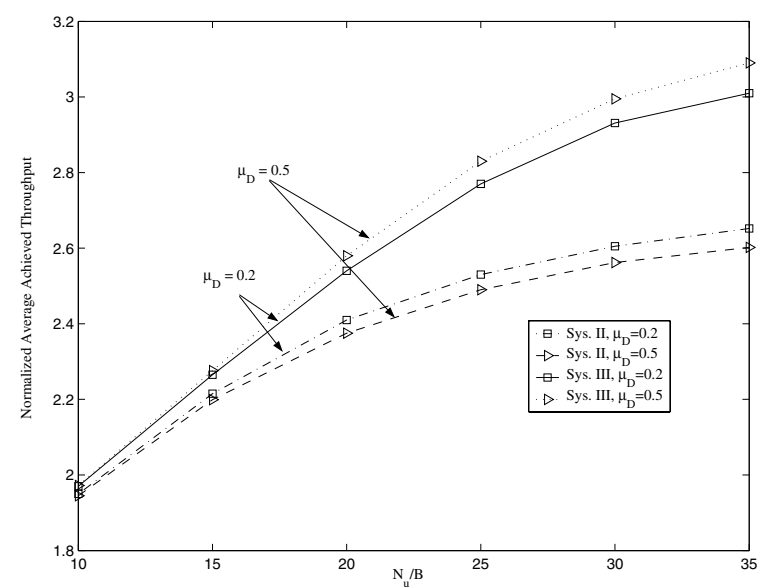

Fig. 4. Normalized average achieved throughput of Systems II and III vs. average number of users in each cell for $\mu_{D}=0.2,0.5$.

achieved. This is due to exploiting the soft-capacity and multiaccess point diversity in System III.

The effect of the proposed joint BS assignment and packet scheduling algorithm on the system throughput is also studied. For simplicity, the average achieved throughput of Systems II and III are normalized by the average achieved throughput of System I. Fig. 4 illustrates the normalized average achieved throughput versus the average number of users in each cell i.e., $N_{u} / B$. In this simulation we consider two user spatial distributions $\left(\mu_{D}=0.2\right.$ and $\left.\mu_{D}=0.5\right)$. The packet arrival rate $\Lambda$ is set to be equal to 5 packets per second. As it can be seen, the average throughput of System III is larger than that of System II. Note that the throughput gain of System II is due to the exploited multi-user diversity alone. The difference between the throughput gains of Systems II and III indicates the gain due to exploiting multi-access point and soft capacity. This gain is increased by the non-uniformity of the spatial distribution of the users.

\section{CONCLUSION}

In this paper, we propose a joint BS assignment and packet scheduling scheme with polynomial time complexity. Using this method, we exploit multi-user diversity, multi-access-point diversity and soft-capacity all in one shot with an acceptable level of complexity. Simulation results indicates a significant performance improvement in the packet-drop ratio, and the achieved throughput due to utilizing unused radio resources.

\section{ACKNOWLEDGMENT}

This work was supported by Natural Sciences and Engineering Research Council (NSERC) of Canada.

\section{REFERENCES}

[1] P. Viswanath, D. Tse, and R. Larioa, "Opportunistic beamforming using dumb antennas," IEEE Transactions on Information Theory, vol. 48, no. 6, pp. 1277-1294, June 2002.

[2] 3GPP, "Technical specification group radio access network. High Speed Downlink Packet Access: overall UTRAN description," $3 G P P$ TR 25.855v5.0.0, 2002.

[3] 3GPP2, "Introduction to 1xEV-DV air interface," 2002.

[4] H. Holma and A. Toskala, WCDMA for UMTS: Radio Access for Third Generation Mobile Communications. John Wiley and Sons, 2000.

[5] K. Navaie and H. Yanikomeroglu, "Optimal downlink resource allocation for non-realtime traffic in cellular CDMA/TDMA networks," to appear in IEEE Communications Letters, 2006.

[6] F. P. Kelly, "Charging and rate control for elastic traffic," European Transactions on Telecommunications, vol. 8, pp. 33-37, January 1997.

[7] K. Navaie, D. Y. Montuno, H. Yanikomeroglu, and Y. Q. Zhao, "Optimal downlink resource allocation for cellular cdma networks," in Adaptation Techniques in Wireless Multimedia Networks, Y. Xiao and W. Li, Eds. Nova Science Publisher, 2006.

[8] C. U. Saraydar, N. B. Mandayam, and D. J. Goodman, "Pricing and power control in a multicell wireless data network," IEEE Journal on Selected Areas in Communications, vol. 19, no. 10, pp. 1883-1892, October 2001.

[9] J. W. Lee, R. R. Mazumdar, and N. B. Shroff, "Joint resource allocation and base-station assignment for the downlink in CDMA networks," to appear in IEEE/ACM Transactions on Networking, February 2006.

[10] X. Liu, E. K. P. Chong, and N. B. Shroff, "Transmission scheduling for efficient wireless network utilization," in Proc. of IEEE INFOCOM'O1, vol. 2, pp. 776-785, April 2001.

[11] R. D. Yates and C. Y. Huang, "Integrated power control and base station assignment," IEEE Transactions on Vehicular Technology, vol. 44, no. 3 , pp. 638-644, August 1995.

[12] M. Shabani and K. Navaie, "Joint pilot power adjustment and base station assignment for data traffic in cellular CDMA networks," in Proc. of IEEE/Sarnoff Symp., pp. 179-183, 2004.

[13] M. Shabani, K. Navaie, and E. S. Sousa, "Downlink resource allocation for data traffic in heterogeneous cellular CDMA networks," in Proc. of IEEE ISCC'04, vol. 1, pp. 436-441, 2004.

[14] C. Makaya and S. Aissa, "Joint scheduling and base station assignment for CDMA packet data networks," in Proc. of IEEE VTC 2003-Fall, vol. 3, pp. 1693-1697, 2003.

[15] K. Navaie and A. R. Sharafat, "A framework for UMTS air interface analysis," Canadian Journal of Electrical and Computer Engineering, vol. 28, no. 2, pp. 113-129, July/October 2003.

[16] Y. Liu and E. W. Knightly, "Opportunistic fair scheduling over multiple wireless channels," in Proc. of IEEE INFOCOM'03, vol. 2, pp. 1106$1115,2003$.

[17] S. Shakkottai and A. Stolyar, "Scheduling for multiple flows sharing a time-varying channel: The exponential rule," in American Mathematical Society Translations, A volume in memory of $F$. Karpelevich, ser. 2 , Y. M. Suhov, Ed., vol. 207, pp. 185-202, 2002.

[18] H. Keller, U. Pferschy, and D. Pisinger, Knapsack Problems. SpringerVerlag, 2004.

[19] H. Everett, "Generalized Lagrange multiplier method for solving problems of optimum allocation of resources," Operation Research, vol. 11, no. 3, pp. 399-417, May-June 1963. 